\title{
Mast Cells Modulate Interleukin-4 Production Independently of Interferon-gamma Generation by Invariant Natural Killer T Cells through the Suppression of Notch Signaling
}

\author{
Daisuke Kurihara, Nobuyuki Fukuishi, ${ }^{*}$ Kazue Kadota, Hirofumi Hamano, Hayato Teruya, \\ Nobuaki Matsui, and Masaaki Akagi \\ Department of Pharmacology, Faculty of Pharmaceutical Sciences, Tokushima Bunri University, Nishihama Bouji 180, Yamashiro-cho, \\ Tokushima, 770-8514, Japan
}

(Received November 25, 2010; Accepted December 4, 2010; Published online December 10, 2010)

The spatial contact of immune cells is predominant machinery for the modulation of local inflammatory sites as much as humoral regulation is for inflammation. In this study, we investigated the role of mast cells in the regulation of invariant natural killer T cells (iNKTs) functions. Co-cultivation of bonemarrow derived mouse mast cells (BMMCs) with purified and stimulated mouse iNKTs caused marked suppression of interleukin (IL)-4 but not interferon (IFN)- $\gamma$ production in iNKTs. Suppression was accompanied by down-regulation of IL-4 mRNA expression. The Notch intracellular cytoplasmic domain in iNKTs was also completely diminished by co-cultivation with BMMCs. Suppressed IL-4 production was recovered when iNKTs were separately co-cultured with BMMCs using the transwell system. Furthermore, co-culture of iNKTs with activated BMMCs elicited partial suppression of IL-4 production. These findings suggest that mast cells modulate the function of iNKTs via the inhibition of Notch signaling.

Key words — mast cell, invariant natural killer T cell, Notch signaling, cell-cell contact

\section{INTRODUCTION}

Mast cells (MCs) are pivotal effector cells in allergic responses and are involved in defense mecha-

\footnotetext{
*To whom correspondence should be addressed: Department of Pharmacology, Faculty of Pharmaceutical Sciences, Tokushima Bunri University, Nishihama Bouji 180, Yamashiro-cho, Tokushima, 770-8514, Japan. Tel.: +81-88-622-9611; Fax: +81-88-655-3051; E-mail: nobuf@ph.bunri-u.ac.jp
}

nisms at local sites of infection. ${ }^{1,2)}$ In such roles, MCs control immune cell fate by generating cytokines, chemical mediators, and cell-cell contact, thereby orchestrating the immune response. ${ }^{3)}$ Physical cell-cell contact, in particular, is one of the central mechanisms for regulation of immune cell differentiation and proliferation. For example, spatial contact of MCs with $\mathrm{T}$ lymphocytes is crucial for the activation of T cells. ${ }^{4-6)}$ MCs sustain the B cell expansion and drive the development of humoral immune responses through cluster of differentiation (CD) 40-CD40 ligand (CD40L) interaction. ${ }^{7)}$ Moreover, MCs inhibit regulatory $\mathrm{T}$ cell suppression and functional $\mathrm{T}$ helper (Th) 17 cell modulation by the surface expressed OX40 ligand (OX40L), ${ }^{8)}$ such cell-cell contact has a potential role in pathogenesis of experimental autoimmune encephalomyelitis and neutrophilic hyperreactivity. ${ }^{8)}$ Invariant natural killer T cells (iNKTs) are innate-like T lymphocytes characterized by promptly generating large amounts of both interferon (IFN)- $\gamma$ and interleukin (IL)-4 following stimulation, ${ }^{9,10)}$ thereby controlling cell fate such as differentiation to $\mathrm{T}$ cells and functioning as dendritic cells. ${ }^{11)}$ This capability of iNKTs is pivotal for tolerance or inflammation at local sites. ${ }^{11,12)}$ Although such participation in regulation of local immune responses through quick and massive cytokine production is well established, control mechanisms for Th1 or Th2 cytokine production in iNKTs are poorly understood. Here, we present control mechanisms of Th1/Th2 cytokine production in iNKTs by MCs through cell-cell contact. This is the first report of modulation of cytokine production by physical interaction with iNKTs. 


\section{MATERIALS AND METHODS}

Mice — C57BL/6 mice (Japan SLC, Shizuoka, Japan) were used. All animal studies were approved by the Animal Care and Use Committee of the Faculty of Pharmaceutical Sciences, Tokushima Bunri University (Tokushima, Japan).

Reagents — Fluorescein isothiocyanate (FITC) anti-T cell receptor (TCR) $\beta$ (clone: H57-597), phycoerythrin (PE) anti-CD19 (clone: 1D3), and allophycocyanine (APC) anti-mouse IgG1 monoclonal antibodies (mAbs) were purchased from BD Biosciences (San Diego, CA, U.S.A.). Anti-mouse $\mathrm{CD} 3 \varepsilon$ (clone: $2 \mathrm{C} 11$ ), FITC anti-mouse Fc $\varepsilon$ RI $\alpha$ (clone: MAR-1), and PE anti-mouse c-Kit/CD117 (clone: 2B8) Abs were obtained from eBioscience (San Diego, CA, U.S.A.) and BD Biosciences. Anti-dinitrophenyl (DNP) IgE and DNP-human serum albumin (HSA) were purchased from Sigma (St. Louis, MO, U.S.A.). Alphagalactosylceramide ( $\alpha$-GalCer) (KRN7000; Kirin Brewer, Gunma, Japan) was dissolved in $0.5 \%$ Tween 20-phosphate-buffered saline (PBS) with sonication for use. $\gamma$-Secretase inhibitors, $\mathrm{N}-[\mathrm{N}$ (3,5-Difluorophenylacetyl)-L-alanyl]-L-2-phenylglycine t-butyl ester (DAPT) and L-685,458 (Peptide Institute, Osaka, Japan), were dissolved in dimethyl sulfoxide (DMSO) for use. The concentrations of these reagents are cited in Dovey and Kang. ${ }^{13,14)}$ Mouse recombinant IL-3 was obtained from Miltenyi Biotech GmbH (Bergisch Gladbach, Germany).

Flow Cytometry — iNKTs were detected by FITC anti-TCR $\beta$ and APC $\alpha$-GalCer-loaded CD1d:Ig (BD Biosciences) as previously described. ${ }^{15)}$ BMMCs were incubated with FITC anti-mouse FceRI $\alpha$ and PE anti-mouse CD117. For detection of Notch-1, anti-mouse Notch-1 mAb (clone: A6, Thermo Scientific, San Jose, CA, U.S.A.) and PE anti-mouse IgG Ab (BioLegend, San Diego, CA, U.S.A.) were used. For detection of Notch ligands, anti-Delta like (DLL) 1 (clone: HMD1-5), DLL4 (clone: HMD4-1), Jagged-1 (clone: HMJ1-29), and Jagged-2 (clone: HMJ2-1) were used (eBioscience and BioLegend).

Isolation of iNKTs - Initially, mice were intraperitoneally injected with $5 \mu \mathrm{g} \alpha$-GalCer. After 3 days, whole spleen cells were prepared. iNKTs were positively selected from the B cell-depleted fraction by APC $\alpha$-GalCer/CD1d:Ig and anti-APC MicroBeads (Miltenyi Biotech) as described. ${ }^{15,16)}$ The purity of the enriched cells was $>95 \%$ iNKTs.
Mouse BMMCs — BMMCs were obtained by culturing mouse bone marrow as described. ${ }^{17,18)}$ The purity of cultured cells was $>97 \%$ BMMCs. To stimulate cells, BMMCs were preincubated with $250 \mathrm{ng} / \mathrm{ml}$ anti-DNP IgE overnight in a $\mathrm{CO}_{2}$ incubator, and then stimulated with $100 \mathrm{ng} / \mathrm{ml}$ DNP- HSA at $37^{\circ} \mathrm{C}$.

Co-culture of iNKTs with BMMCs — Purified iNKTs and BMMCs were suspended in complete RPMI1640 containing $50 \mu \mathrm{M}$ 2-mercaptoethanol (Kanto Chemical, Tokyo, Japan). iNKTs and BMMCs, both at $2 \times 10^{5}$, were co-cultured in 96well plates with $10 \mu \mathrm{g} / \mathrm{ml}$ plate-coated anti-mouse $\mathrm{CD} 3 \varepsilon$ at $37^{\circ} \mathrm{C}$ for $6-12 \mathrm{hr}$. After co-culture, the supernatant was obtained for the cytokine assay. To prevent cell-cell contact, cell culture inserts (transwells; pore size: $0.2 \mu \mathrm{m}$, Nunc, Rochester, NY, U.S.A.) were used.

Cytokine Measurement — The levels of IL-4 and IFN- $\gamma$ in the culture supernatants were assayed by Flowcytomix (Bender MedSystems, Hinckley Road Burlingame, CA, U.S.A.) according to the manufacturer's instruction.

Real-time PCR — cDNA was prepared from total RNA using poly (dT) and SuperScript II (Invitrogen, Carlsbad, CA, U.S.A.). mRNA expression of IL-4 and glyceraldehyde-3-phosphate dehydrogenase (GAPDH) was measured by quantitative real-time PCR using TaqMan Gene Expression Assays (Applied Biosystems, Bedford, MA, U.S.A.).

Immunoblotting — Cells were lysed in LDS sample buffer (Invitrogen) containing protease inhibitor cocktails. Samples were analyzed by western blotting. For detection of Notch intracellular domain-1 (NICD-1), anti-Notch-1 Ab (clone: mN1A, eBioscience) was used. Enhanced chemiluminescence (ECL) anti-mouse IgG-horseradish peroxidase (HRP, GE Healthcare, Piscataway, NJ, U.S.A.) and Immobilon Western HRP (Millipore, Bedford, MA, U.S.A.) were used.

Statistical Analysis — Results are expressed as means \pm S.E. Statistical significance of differences was determined by a two-tailed unpaired Student's $t$-test. $P$ values less than 0.05 were considered significant.

\section{RESULTS}

\author{
BMMCs Suppress IL-4 Production of iNKT \\ Cells in Co-coculture
}

iNKTs are known to produce a massive amount 
A
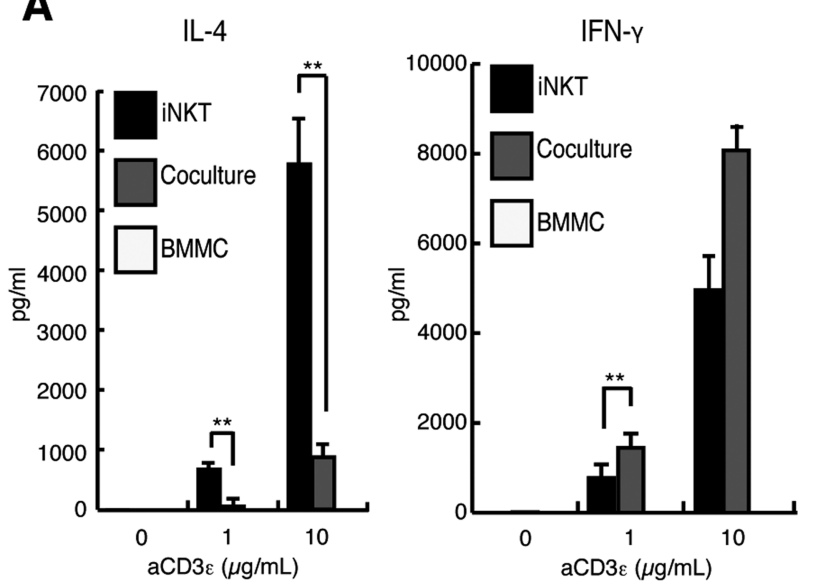

B

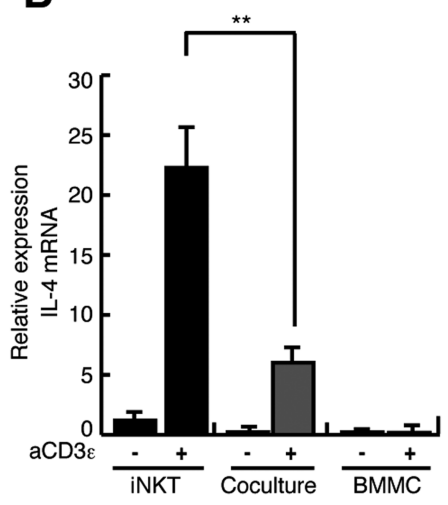

Fig. 1. Cytokine Production and mRNA Expression Rate in iNKTs

A, iNKTs were cultured with or without equal concentration of BMMCs in 96-well plates bound with anti-CD3 $\varepsilon$ for $12 \mathrm{hr}$. The concentrations of IFN- $\gamma$ and IL- 4 in the culture supernatants were measured by Flowcytomix. Data are the means \pm S.E. obtained from three independent experiments. B, IL-4 mRNA levels measured by realtime PCR. Data are the means \pm S.E. of three independent experiments. ${ }^{* *} p<0.01$.

of both IL-4 and IFN- $\gamma$ following activation at the site of inflammation. ${ }^{19-21)}$ These cytokines regulate local immune responses. ${ }^{22,23)}$ We therefore investigated the effect of BMMCs on cytokine production by activated iNKTs. Anti-CD3 $\varepsilon$ stimulation led to IL-4 and IFN- $\gamma$ production in iNKTs. Notably, IL-4 production considerably decreased when activated iNKTs were co-cultured with BMMCs (Fig. 1A) which was accompanied by a downregulation of mRNA expression (Fig. 1B) IFN- $\gamma$ production appeared to slightly increase in the coculture (Fig. 1A).

\section{Cell-cell Contact Is the Important for the Func- tion of BMMCs}

The functions of immune cells are known to

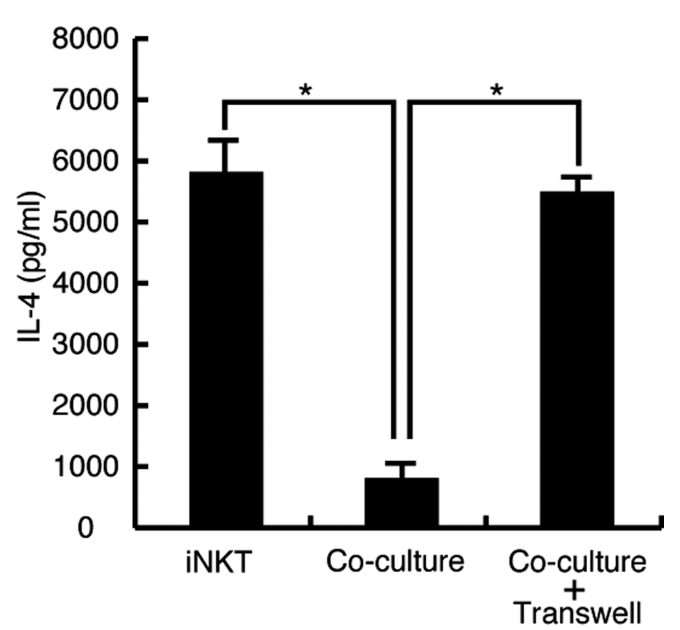

Fig. 2. Spatial Contact with BMMCs Is Essential for Suppression of IL-4 Production in iNKTs

To prevent cell-to-cell interaction, BMMCs were placed in a transwell and cultured with iNKTs on 96-well plates for $12 \mathrm{hr}$. The supernatants were collected and secreted IL-4 was measured. Data are the means \pm S.E. of three independent experiments. ${ }^{*} p<0.05$.

be modulated by spatial cell-to-cell contact besides the humoral factors. ${ }^{24)}$ We thus examined the mechanisms of reduced IL-4 generation in iNKT by BMMCs co-cultivation, using transwell system. Prevention of physical contact between iNKTs and BMMCs by the transwell completely recovered suppression of IL-4 production (Fig. 2). These findings indicate that the reduction of IL-4 production by BMMCs requires cell-cell contact.

\section{Notch-1 Signaling Is Involved in the IL-4 Pro- duction in iNKT Cells}

Notch signaling has been indicated as a pivotal mechanism for IL-4 production in $\mathrm{CD}^{+} \mathrm{T}$ cells ${ }^{25,26)}$ and NKT cells. ${ }^{27)}$ Okamoto et al. showed the involvement of Notch in IL-4 production and Th2 proliferation. ${ }^{28)}$ The interaction of Notch receptors and their ligands causes release of NICD into the cytosol, which translocates NICD to the nucleus. ${ }^{29)}$ In the nucleus, NICD binds to transcription factors and up-regulates the target genes. ${ }^{26,29)}$

We next focused on Notch signaling in the production of IL-4 in activated iNKTs. The surface expression of Notch-1 as well as its ligands was validated on iNKTs (Fig. 3A). NICD was also observed in activated iNKTs, but was completely diminished following co-culturing of activated iNKTs with BMMCs (Fig. 3C). DAPT and L-685,458, typical $\gamma$-secretase inhibitors, mildly depressed the Notch signaling (Fig. 3C), in addition to slightly decreasing anti-CD3 $\varepsilon$-induced IL-4 production in iNKTs 
A
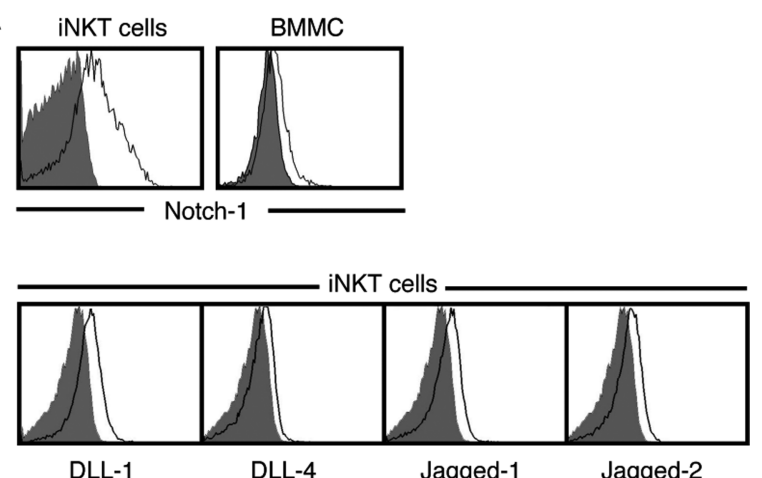

B

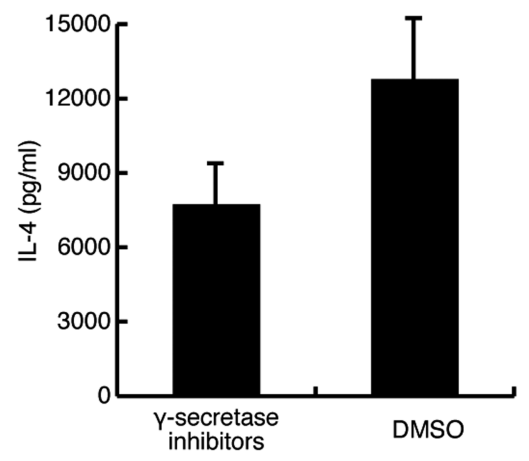

C

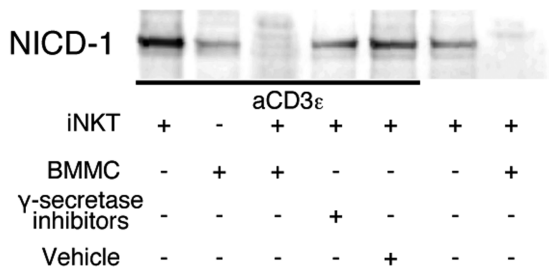

Fig. 3. Notch Signaling Is Involved in the Suppression of IL-4 Production in iNKTs

A, Expression of Notch-1 and Notch ligands in iNKTs or BMMCs. Result of one representative experiment out of two is shown. B, DAPT and L-685,458, $\gamma$-secretase inhibitors, partly blocked IL-4 production. Results shown are from three independent experiments. C, Observations of NICD in iNKTs. iNKTs and BMMCs, both at $1 \times 10^{6}$, were co-cultured for $6 \mathrm{hr}$ and analyzed by western blotting. Result shown is one representative experiment out of three.

(Fig. 3B). These findings suggest that iNKTs constitutively transactivate through surface-expressed Notch and its ligand, signaling that may be pivotal for IL-4 production on activated iNKTs.

\section{IgE-stimulated BMMCs Partly Reduce the In- hibiting Function}

We thus further investigated the impact of activated MCs on the inhibition of IL-4 production in activated iNKTs. Following a lapse of $5 \mathrm{~min}$ or $4 \mathrm{hr}$, IgE-cross-linked BMMCs were incubated
A

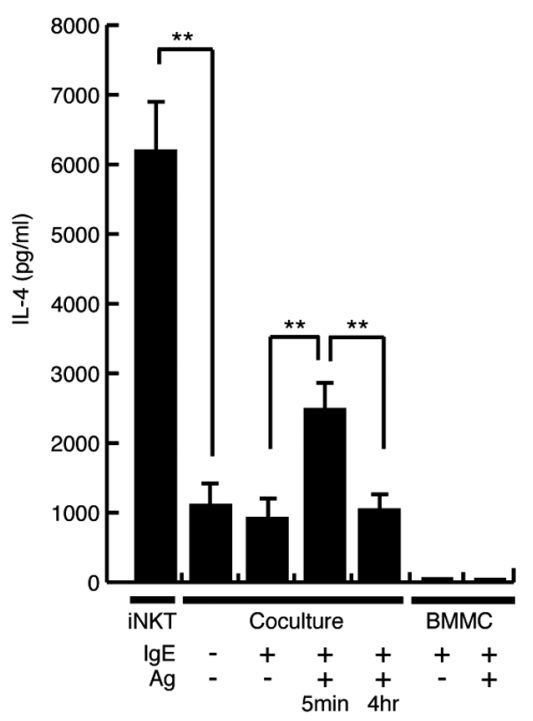

B

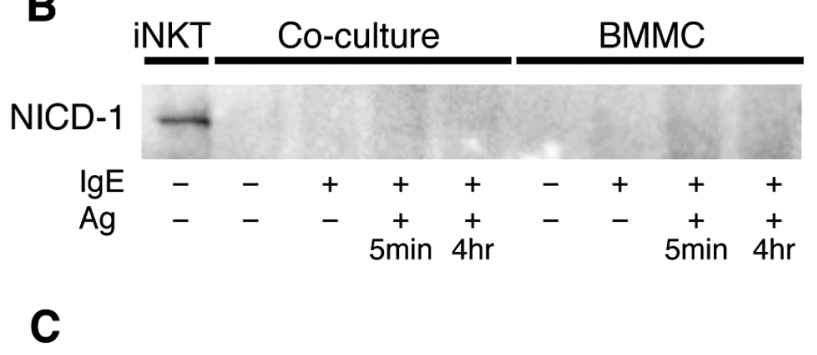

NICD-1

$5 \mathrm{~min} 4 \mathrm{hr}$

Fig. 4. Relationship between IL-4 Production and NICD in iNKTs

A, iNKT were co-cultured with IgE-sensitized BMMCs or nonsensitized BMMCs which were stimulated by DNP-HSA for $5 \mathrm{~min}$ or $4 \mathrm{hr}$, respectively. Cultured supernatant was used for cytokine measurement. Data are the means \pm S.E. of three independent experiments. ${ }^{* *} p<0.01$. B, Cells were collected after $6 \mathrm{hr}$ co-culture and NICD was detected by western blotting. Result shown is one representative experiment out of three. C, Cells were collected after 15-min co-culture, and NICD was detected by western blotting. Result shown is one representative experiment out of three.

with iNKTs in an anti-CD3e-coated culture dish for $12 \mathrm{hr}$. IL-4 production was markedly diminished in co-cultures of iNKTs with BMMCs stimulated for $4 \mathrm{hr}$ or with non-stimulated BMMCs, but this inhibition was partially recovered in cocultures of iNKTs with BMMCs stimulated after 5 min (Fig. 4A). NICD was not detected even after $6 \mathrm{hr}$ in iNKT co-cultures with BMMCs stimulated for $5 \mathrm{~min}$ or $4 \mathrm{hr}$ (Fig. 4B). On the other hand, after $15 \mathrm{~min}$ in iNKT co-cultures, NICD was slightly detected with BMMCs stimulated at $4 \mathrm{hr}$ and clearly detected with BMMCs stimulated for $5 \mathrm{~min}$ (Fig. 4C). These findings indicate that al- 
though 5-min stimulation of BMMCs did not affect Notch signaling, 4-hr stimulation inhibited it.

\section{DISCUSSION}

Type 2 helper T cells act as the "manufacturing equipment" of Th2-type cytokine such as IL4 , IL-5, and IL-13, ${ }^{30,31)}$ and these cytokines control the pathology of the local site of the inflammation. ${ }^{31)}$ This type of $\mathrm{T}$ cell is especially pivotal for the elimination of the non-toxic intruder or parasites inversions, ${ }^{32)}$ and also plays a key role in the disease onset of allergic inflammation such as allergic asthma. ${ }^{33)}$ Although Th2 fate specification and commitment from naïve $\mathrm{T}$ lymphocytes require "primarily IL-4" assistance, ${ }^{31)}$ little is known about the source of the primarily IL-4 on Th2 responses. Whereas, it is widely accepted that activated iNKTs quickly generate a massive amount of IL-4 and IFN$\gamma,{ }^{20,21)}$ and therefore activated iNKTs are the leading candidate of primarily IL-4. ${ }^{31)}$ We indicated in this paper that MCs could modulate cytokines generation on activated iNKTs by physically cell-tocell contact mechanism, and this is the first report which suggests that MCs can selectively modulate "primarily" IL-4 production in iNKTs. The crucial roles of MCs surface expressed OX40 on T cell proliferation, ${ }^{4,5)}$ differentiation, ${ }^{8)}$ and cytokine generation $^{5)}$ have been recently reported. As well, the importance of inducible T-cell costimulator (ICOS) on iNKTs functions has also been indicated. ${ }^{34)} \mathrm{We}$ also investigated the participation of OX40-OX40L or ICOS-ICOS ligand (ICOSL) axes in the suppression of IL-4 production by BMMCs, using both anti-OX40L and/or anti ICOSL neutralizing antibodies. However, both receptor-ligand interactions were not involved in the suppression of IL-4 (data not shown). Whereas, neutralizing antibodies for DLL1, DLL4, Jagged-1, and Jagged-2, which are known to Notch ligands, did also not affect the down-regulation of IL-4 (data not shown), although incubation of activated iNKTs with BMMCs caused the complete elimination of NICD. Moreover, the diminution of IL- 4 production by BMMCs was partly canceled when activated iNKTs were cocultured with just activated BMMCs. These observations indicate that the mechanisms of the IL-4 suppression by BMMCs is involved in some unidentified molecules that inhibit Notch or NICD. As FceRI cross-linking on the surface of MCs is known to induce MCs membrane perturbing, ${ }^{35)}$ the partial blocking of suppressed IL-4 production by activated BMMCs may be a result of partial cancellation of Notch or NICD inhibition on the surface of BMMCs by activation-caused membrane disturbance. The NICD expression in BMMCs also seemed to be extinguished when iNKTs were co-cultivated with BMMCs. Co-cultivation of iNKTs might also affect the function of MCs. However, the target inhibitory molecules of the Notch and/or NICD on the surface of BMMCs, which also may affect BMMCs function, could not be identified in this study. Further studies are needed to clarify this point.

The observation of NICD in non-activated iNKTs and the cancellation of IL-4 suppression by activated BMMCs are intriguing because these observations suggest that iNKTs constitutively pre-transactivate through surface-expressed Notch-ligand signaling, and that IL-4 production in activated iNKTs is regulated by the status of the other cells such as MCs. In other words, these results imply that cytokines generation pattern on activated iNKTs is able to be controlled by both the local density of iNKTs and the difference of co-cumulative immune cells class. The variety of iNKTs activation may be closely associated with the pathology of the local site of inflammation.

In conclusion, this study clarified that the inhibition of IL-4 production by co-culture is closely related with the state of MCs. That is, MCs appear to control IL-4 production in iNKTs at local inflammatory sites through Notch signaling. This is the first report demonstrating a regulatory role of MCs in the production of cytokines in iNKTs

Acknowledgements This work was supported by a grant from Tokushima Bunri University.

\section{REFERENCES}

1) Galli, S. J. and Tsai, M. (2010) Mast cells in allergy and infection: versatile effector and regulatory cells in innate and adaptive immunity. Eur. J. Immunol., 40, 1843-1851.

2) Mekori, Y. A. and Metcalfe, D. D. (2000) Mast cells in innate immunity. Immunol. Rev., 173, 131-140.

3) Kalesnikoff, J. and Galli, S. J. (2008) New developments in mast cell biology. Nat. Immunol., 9, 12151223.

4) Kashiwakura, J., Yokoi, H., Saito, H. and Okayama, Y. (2004) T cell proliferation by direct cross-talk between OX40 ligand on human mast cells and OX40 
on human T cells: comparison of gene expression profiles between human tonsillar and lung-cultured mast cells. J. Immunol., 173, 5247-5257.

5) Nakae, S., Suto, H., Iikura, M., Kakurai, M., Sedgwick, J. D., Tsai, M. and Galli, S. J. (2006) Mast cells enhance $\mathrm{T}$ cell activation: importance of mast cell costimulatory molecules and secreted TNF. J. Immunol., 176, 2238-2248.

6) Hershko, A. Y. and Rivera, J. (2010) Mast cell and $\mathrm{T}$ cell communication; amplification and control of adaptive immunity. Immunol. Lett., 128, 98-104.

7) Merluzzi, S., Frossi, B., Gri, G., Parusso, S., Tripodo, C. and Pucillo, C. (2010) Mast cells enhance proliferation of B lymphocytes and drive their differentiation toward IgA-secreting plasma cells. Blood, 115, 2810-2817.

8) Piconese, S., Gri, G., Tripodo, C., Musio, S., Gorzanelli, A., Frossi, B., Pedotti, R., Pucillo, C. E. and Colombo, M. P. (2009) Mast cells counteract regulatory T-cell suppression through interleukin-6 and OX40/OX40L axis toward Th17-cell differentiation. Blood, 114, 2639-2648.

9) Kronenberg, M. (2005) Toward an understanding of NKT cell biology: progress and paradoxes. Аnпи. Rev. Immunol., 23, 877-900.

10) Kronenberg, M. and Gapin, L. (2002) The unconventional lifestyle of NKT cells. Nat. Rev. Immunol., 2, 557-568.

11) Tupin, E., Kinjo, Y. and Kronenberg, M. (2007) The unique role of natural killer $\mathrm{T}$ cells in the response to microorganisms. Nat. Rev. Microbiol., 5, 405-417.

12) Emoto, M. and Kaufmann, S. H. (2003) Liver NKT cells: an account of heterogeneity. Trends Immunol., 24, 364-369.

13) Dovey, H. F., John, V., Anderson, J. P., Chen, L. Z., de Saint Andrieu, P., Fang, L. Y., Freedman, S. B., Folmer, B., Goldbach, E., Holsztynska, E. J., Hu, K. L., Johnson-Wood, K. L., Kennedy, S. L., Kholodenko, D., Knops, J. E., Latimer, L. H., Lee, M., Liao, Z., Lieberburg, I. M., Motter, R. N., Mutter, L. C., Nietz, J., Quinn, K. P., Sacchi, K. L., Seubert, P. A., Shopp, G. M., Thorsett, E. D., Tung, J. S., Wu, J., Yang, S., Yin, C. T., Schenk, D. B., May, P. C., Altstiel, L. D., Bender, M. H., Boggs, L. N., Britton, T. C., Clemens, J. C., Czilli, D. L., Dieckman-McGinty, D. K., Droste, J. J., Fuson, K. S., Gitter, B. D., Hyslop, P. A., Johnstone, E. M., Li, W. Y., Little, S. P., Mabry, T. E., Miller, F. D. and Audia, J. E. (2001) Functional gamma-secretase inhibitors reduce beta-amyloid peptide levels in brain. J. Neurochem., 76, 173-181.

14) Kang, J. H., Kim, B. S., Uhm, T. G., Lee, S. H., Lee, G. R., Park, C. S. and Chung, I. Y. (2009) Gamma- secretase inhibitor reduces allergic pulmonary inflammation by modulating Th1 and Th2 responses. Am. J. Respir. Crit. Care Med., 179, 875-882.

15) Watarai, H., Nakagawa, R., Omori-Miyake, M., Dashtsoodol, N. and Taniguchi, M. (2008) Methods for detection, isolation and culture of mouse and human invariant NKT cells. Nat. Protoc., 3, 70-78.

16) Godfrey, D. I., MacDonald, H. R., Kronenberg, M., Smyth, M. J. and Van Kaer, L. (2004) NKT cells: what's in a name? Nat. Rev. Immunol., 4, 231-237.

17) Razin, E., Ihle, J. N., Seldin, D., Mencia-Huerta, J. M., Katz, H. R., LeBlanc, P. A., Hein, A., Caulfield, J. P., Austen, K. F. and Stevens, R. L. (1984) Interleukin 3: A differentiation and growth factor for the mouse mast cell that contains chondroitin sulfate $\mathrm{E}$ proteoglycan. J. Immunol., 132, 1479-1486.

18) Mekori, Y. A., Oh, C. K. and Metcalfe, D. D. (1993) IL-3-dependent murine mast cells undergo apoptosis on removal of IL-3. Prevention of apoptosis by c-kit ligand. J. Immunol., 151, 3775-3784.

19) Bendelac, A., Rivera, M. N., Park, S. H. and Roark, J. H. (1997) Mouse CD1-specific NK1 T cells: development, specificity, and function. Annu. Rev. Immunol., 15, 535-562.

20) Taniguchi, M., Harada, M., Kojo, S., Nakayama, T. and Wakao, H. (2003) The regulatory role of Valpha14 NKT cells in innate and acquired immune response. Annu. Rev. Immunol., 21, 483-513.

21) Stetson, D. B., Mohrs, M., Reinhardt, R. L., Baron, J. L., Wang, Z. E., Gapin, L., Kronenberg, M. and Locksley, R. M. (2003) Constitutive cytokine mRNAs mark natural killer (NK) and NK T cells poised for rapid effector function. J. Exp. Med., 198, 1069-1076.

22) Kronenberg, M. (2005) Toward an understanding of NKT cell biology: progress and paradoxes. Anпи. Rev. Immunol., 23, 877-900.

23) Akbari, O., Stock, P., Meyer, E., Kronenberg, M., Sidobre, S., Nakayama, T., Taniguchi, M., Grusby, M. J., DeKruyff, R. H. and Umetsu, D. T. (2003) Essential role of NKT cells producing IL-4 and IL13 in the development of allergen-induced airway hyperreactivity. Nat. Med., 9, 582-588.

24) Montoya, M. C., Sancho, D., Vicente-Manzanares, M. and Sanchez-Madrid, F. (2002) Cell adhesion and polarity during immune interactions. Immunol. Rev., 186, 68-82.

25) Amsen, D., Blander, J. M., Lee, G. R., Tanigaki, K., Honjo, T. and Flavell, R. A. (2004) Instruction of distinct CD4 $\mathrm{T}$ helper cell fates by different notch ligands on antigen-presenting cells. Cell, 117, 515526.

26) Amsen, D., Antov, A., Jankovic, D., Sher, A., 
Radtke, F., Souabni, A., Busslinger, M., McCright, B., Gridley, T. and Flavell, R. A. (2007) Direct regulation of Gata3 expression determines the T helper differentiation potential of Notch. Immunity, 27, 8999.

27) Tanaka, S., Tsukada, J., Suzuki, W., Hayashi, K., Tanigaki, K., Tsuji, M., Inoue, H., Honjo, T. and Kubo, M. (2006) The interleukin-4 enhancer CNS2 is regulated by Notch signals and controls initial expression in NKT cells and memory-type CD4 T cells. Immunity, 24, 689-701.

28) Okamoto, M., Matsuda, H., Joetham, A., Lucas, J. J., Domenico, J., Yasutomo, K., Takeda, K. and Gelfand, E. W. (2009) Jagged1 on dendritic cells and Notch on CD4+ T cells initiate lung allergic responsiveness by inducing IL-4 production. J. Immunol., 183, 2995-3003.

29) Tsukumo, S. and Yasutomo, K. (2004) Notch governing mature $\mathrm{T}$ cell differentiation. J. Immunol., 173, 7109-7113.

30) Fallon, P. G., Jolin, H. E., Smith, P., Emson, C. L., Townsend, M. J., Fallon, R. and McKenzie, A. N. (2002) IL-4 induces characteristic Th2 responses even in the combined absence of IL-5, IL-9, and IL13. Immunity, 17, 7-17.

31) Barrett, N. A. and Austen, K. F. (2009) Innate cells and $\mathrm{T}$ helper 2 cell immunity in airway inflammation. Immunity, 31, 425-437.

32) Anthony, R. M., Rutitzky, L. I., Urban, J. F., Jr., Stadecker, M. J. and Gause, W. C. (2007) Protective immune mechanisms in helminth infection. Nat. Rev. Immunol., 7, 975-987.

33) Holgate, S. T. and Polosa, R. (2008) Treatment strategies for allergy and asthma. Nat. Rev. Immunol., 8, 218-230.

34) Akbari, O., Stock, P., Meyer, E. H., Freeman, G. J., Sharpe, A. H., Umetsu, D. T. and DeKruyff, R. H. (2008) ICOS/ICOSL interaction is required for CD4+ invariant NKT cell function and homeostatic survival. J. Immunol., 180, 5448-5456.

35) Rosenwasser, L. J., O’Brien, T. and Weyne, J. (2005) Mast cell stabilization and anti-histamine effects of olopatadine ophthalmic solution: a review of pre-clinical and clinical research. Curr. Med. Res. Opin., 21, 1377-1387. 